\title{
Tests on Pretrained Superelastic NiTi Shape Memory Alloy Rods: Towards Application in Self-Centering Link Beams
}

\author{
Xian Xu $\left(\mathbb{D},{ }^{1}\right.$ Guangming Cheng, ${ }^{1}$ and Junhua Zheng ${ }^{2}$ \\ ${ }^{1}$ Department of Civil Engineering, Zhejiang University, 866 Yuhangtang Road, Hangzhou, Zhejiang 310058, China \\ ${ }^{2}$ Institute of Urban Construction, Hangzhou Polytechnic, 198 Gaoke Road, Hangzhou, Zhejiang 311402, China \\ Correspondence should be addressed to Xian Xu; xian_xu@zju.edu.cn
}

Received 12 October 2017; Accepted 22 February 2018; Published 1 April 2018

Academic Editor: Stefano Sorace

Copyright (C) 2018 Xian Xu et al. This is an open access article distributed under the Creative Commons Attribution License, which permits unrestricted use, distribution, and reproduction in any medium, provided the original work is properly cited.

\begin{abstract}
Austenitic shape memory alloy has potential applications in self-centering seismic resistant structural systems due to its superelastic response under cyclic tension. Raw austenitic SMA needs proper pretreatments and pretraining to gain a stable superelastic property. In this paper, tests are carried out to investigate the effects of pretraining, pretreatments, loading rate, and strain amplitude on the mechanical performance on austenitic SMA rods with a given size. The tested rods are to be used in a new concept self-centering steel link beam. Customized pretraining scheme and heat treatment are determined through the tests. The effects of loading rate and strain amplitude are investigated. A simplified stress-strain model for the SMA rods oriented to numerical simulations is obtained based on the test results. An example of using the simplified material model in numerical analysis of a self-centering steel link beam is conducted to validate the applicability of the model.
\end{abstract}

\section{Introduction}

Besides the well-known shape memory effect, shape memory alloy at the austenite state exhibits a superelastic effect due to crystallographic transformation between austenite phase and martensite phase under certain conditions [1]. During cyclic loading, the superelastic SMA is able to undergo reversible strain up to $8 \%$ and exhibit flag-shaped stressstrain loops [1]. An amount of energy equivalent to the areas enveloped by the stress-strain loops is dissipated during the cyclic loading. Due to its unique energy dissipation behavior and high fatigue life, SMA has been studied for use as damping device in seismic resistant structures since 1990s [2-6]. In these applications, SMA in wire form is usually used due to its low cost and no need for further machining [7].

Recognizing the similarity between the flag-shaped stress-strain loop exhibited by the superelastic SMA and the flag-shaped hysteresis loop observed in typical selfcentering systems, the superelastic SMA, especially in the form of rods, has recently been studied for use as recentering components in self-centering connections [8-15]. Various SMA-based self-centering connections have been proposed.
For example, T-stub connection using SMA bolts was proposed and experimentally investigated by Abolmaali et al. [8]; a self-centering SMA-bolted beam-to-column connection with an extended end plate was proposed by Ma et al. [9, 10] and experimentally validated by Fang et al. [11]; Speicher et al. developed and experimentally validated an interior beam-tocolumn connection reinforced with SMA tendons [12]; Xu et al. proposed a self-centering link beam using posttensioned SMA rods [14]; and a kind of steel-concrete composite connections equipped with SMA bolts was proposed and experimentally validated [15]. The behavior of these materialbased self-centering systems is heavily dependent on the mechanical properties of the material (i.e., the SMA rods). As a result, the mechanical performance of SMA rods has recently also been attracting considerable attention [16-18]. It has been observed that the mechanical performance of SMA rods is affected by a number of parameters including the size, the heat treatment strategy, the cyclic number, and the loading rate $[16,18]$.

In this paper, tests on SMA rods with a given size are reported. The purpose of the tests is to determine the optimal pretreatments for the SMA rods and extract a simplified 
material model for the SMA rods compatible with available commercial FE packages. The layout of the paper is as follows: Section 2 gives the details of the tests. Then, the results of the tests are analyzed and discussed in Section 3. A simplified material model for the SMA rods under optimal pretreatments is fitted from the corresponding test data. An example of using the simplified material model in numerical study of the self-centering link beam is presented in Section 4. Finally, Section 5 concludes the work.

\section{Test Arrangements}

2.1. Raw SMA Rods and Test Coupons. The raw SMA rods used in this paper were provided by a commercial special metal supplier. The alloy was manufactured in accordance with the Standard Specification for Wrought NickelTitanium Shape Memory Alloys for Medical Devices and Surgical Implants (F2063) issued by ASTM. As reported in the "Certification of Product Quality" provided by the supplier, the weight percentage of nickel, carbon, hydrogen, and oxygen in the alloy are $56.01 \%, 0.009 \%, 0.0006 \%$, and $0.024 \%$, respectively, and the weight percentage of nitrogen in the alloy is below $0.003 \%$. The nominal diameter of the raw SMA rods is $20 \mathrm{~mm}$. The nominal austenite start temperature of them is $-20^{\circ} \mathrm{C}$ which guarantees that they are in austenite state in the room temperature. No heat treatment has been conducted on them by the manufacture. The raw SMA rods are machined to dog-bone-shaped coupons, as shown in Figure 1, to concentrate the deformations in the reduced section. The two ends of a coupon are threaded with a net diameter of $18.15 \mathrm{~mm}$. The net diameter of the reduced section is chosen as $14 \mathrm{~mm}$ which ensures that the ratio between the net diameter of the threaded ends and the net diameter of the reduced section is larger than 1.30 to avoid premature failure at the interface between the threaded ends and the segment with reduced size [11].

To simplify the identifications of the specimens, each specimen is notated by an identifier starting with the diameter of the work section, followed by the heating temperature and duration, and ending with the sequence of heat treatment and machining work. HM and $\mathrm{MH}$ were used to represent heat treatment conducted before machining work and heat treatment conducted after machining work, respectively. For example, specimen 14-350-30-MH indicates that the specimen was machined down to the dog-bone shape with $14 \mathrm{~mm}$ diameter over the working segment and is then heated by $350^{\circ} \mathrm{C}$ for 30 minutes. If there is no heat treatment applied on the specimen, the identifier for it will be $14-\mathrm{N}-\mathrm{N}$.

Nine specimens were used in this study to investigate various characteristics of the rods and the details of them are given in Table 1. 4 heat temperatures evenly ranged from $300^{\circ} \mathrm{C}$ to $450^{\circ} \mathrm{C}$ with the same duration of 30 minutes were adopted in the heat treatment of the specimens. After the heat treatment, the specimens were quenched to room temperature by water. Most of the specimens were machined down to the dog-bone shape before the heat treatment, and only one of them was machined after the heat treatment for comparison study. A specimen without heat treatment was

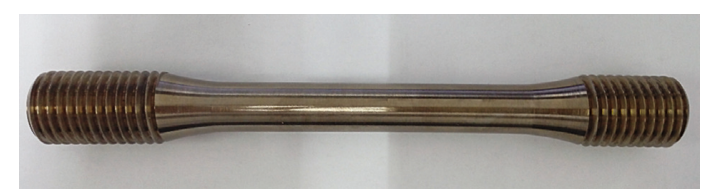

(a)

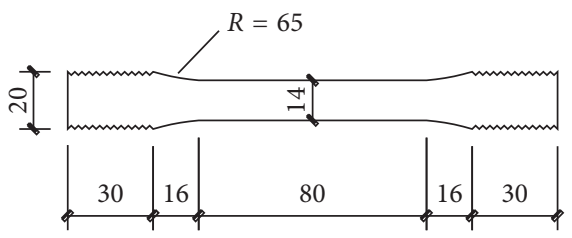

(b)

Figure 1: Test coupon.

also adopted for comparison study. Note that in Table 1 the practical loading protocol used by each specimen indicated a loading protocol code, the definition of which will be given in Section 2.3.

\subsection{Test Setup. A $250 \mathrm{kN}$ INSTRON 8802 servo-controlled} hydraulic test machine was used to perform the tests. Since the SMA specimens were round and hard, the test machine failed to grip them directly by its jaws once the tension reached $70 \mathrm{kN}$. As a result, a set of rectangle steel heads were used to facilitate gripping of the specimens. A $50 \mathrm{~mm}$ length MTS extensometer was used to monitor the strain of the tested specimen. Test setup is shown in Figure 2. In each loading cycle, a strain-controlled criterion was used. The tension and strain data during each test were automatically collected by control computer of the test machine at a sampling frequency of $40 \mathrm{~Hz}$.

2.3. Loading Protocols. Two basic loading protocols LPI and LPII were used in the tests. Loading protocol LPI consists of increasing strain cycles of $1-6 \%$ by increments of $1 \%$, as shown in Figure 3(a). Loading protocol LPII consists of constant strain cycles of 6\%, as shown in Figure 3(b). For loading protocol LPI, the loading was performed at a strain rate of $0.03 \% \mathrm{~s}^{-1}$. For loading protocol LPII, three different strain rates $0.03 \% \mathrm{~s}^{-1}, 0.15 \% \mathrm{~s}^{-1}$, and $0.3 \% \mathrm{~s}^{-1}$ were adopted depending on the cases. For each specimen, the practical loading protocol applied was a combination of the basic ones. To simply indicate the practical loading protocol used for a given specimen, a loading protocol code consisting of the abbreviations of the basic loading protocols and the corresponding parameters (i.e., strain rates and cycle numbers) lined in time order was adopted. For example, loading protocol LP (I-0.03, II-0.15-15, and I-0.03) means that the specimen will be loaded with the basic loading protocol LPI at a strain rate of $0.03 \% \mathrm{~s}^{-1}$, then loaded with the basic loading protocol LPII at a strain rate of $0.15 \% \mathrm{~s}^{-1}$ for 15 cycles, and finally loaded with the basic loading protocol LPI at a strain rate of $0.03 \% \mathrm{~s}^{-1}$ again. The loading protocol codes for each specimen are given in Table 1. 
TABLE 1: Summary of specimens used in this study.

\begin{tabular}{lccc}
\hline Specimen number & Specimen codes & Loading protocols & Numbers \\
\hline 1 & $14-\mathrm{N}-\mathrm{N}$ & LP (I-0.03, II-0.03-25) & 1 \\
2 & $14-300-30-\mathrm{MH}$ & LP (I-0.03, II-0.03-25) & 1 \\
3 & & LP (I-0.03, II-0.03-15, II-0.03-10) & 1 \\
4 & & LP (I-0.03, II-0.03-15, II-0.15-10) & 1 \\
5 & $14-350-30-\mathrm{MH}$ & LP (I-0.03, II-0.03-15, II-0.30-10) & 1 \\
6 & & LP (I-0.03, II-0.03-15, I-0.03) & 1 \\
7 & & LP (I-0.03, II-0.03-25) & 1 \\
8 & $14-400-30-\mathrm{MH}$ & LP (I-0.03, II-0.03-25) & 1 \\
9 & $14-450-30-\mathrm{MH}$ & LP (I-0.03, II-0.03-25) & 1 \\
\hline
\end{tabular}

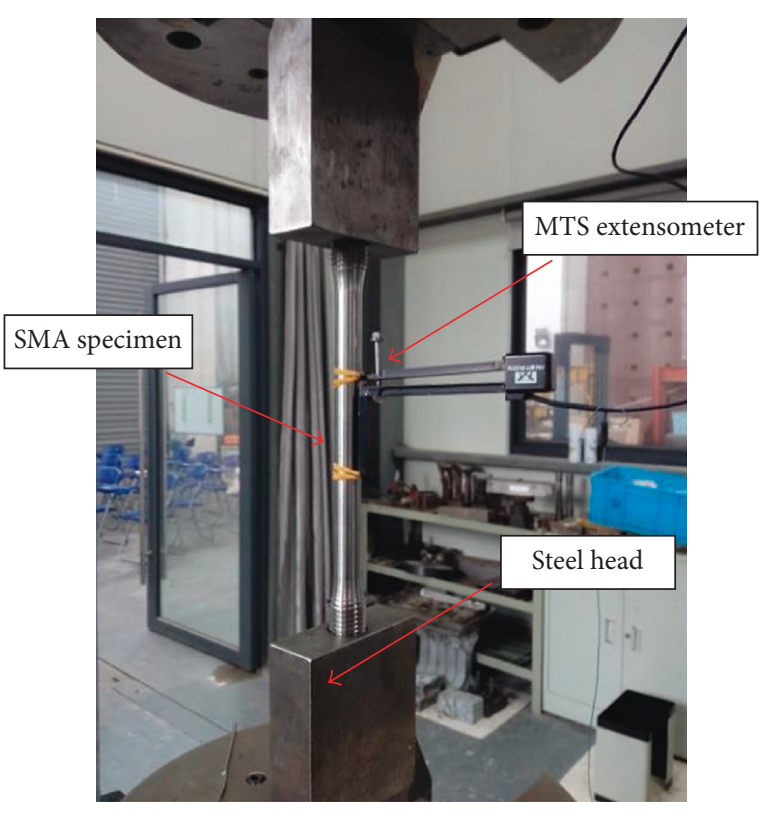

Figure 2: Test setup.

\section{Test Results and Analysis}

3.1. Effect of Loading Cycles and Pretraining Scheme. Previous studies showed that the stress-strain response of SMA rods under each load cycle varied from each other when no prestraining or inadequate pretraining was imposed on the SMA rods [17]. Though a SMA rod without prestraining usually exhibits fatter hysteresis loops during the first several loading cycles, there is nonnegligible increment of residual strain and considerable decrement of the forward transformation stress $\sigma_{\mathrm{Ms}}$ between adjacent cycles.

The forward transformation stress $\sigma_{\mathrm{Ms}}$ is defined as the starting stress for the transformation from austenite to martensite, as shown in Figure 4 which is a diagram for the simplified stress-strain curve of superelastic SMA material. While for the real stress-strain response of superelastic SMA material, as shown in Figure 5, the starting point for the transformation from austenite to martensite is not as clear as that in the simplified curve. As a result, a conditional point was defined as the starting point for the transformation from austenite to martensite (Figure 5). The stress-strain data were fitted to a high order smooth curve, and the point with maximum curvature on the loading segment was defined as the forward transformation stress $\sigma_{\mathrm{Ms}}$. Accordingly, elastic modulus $E_{\mathrm{A}}$ of the SMA material was defined as the tangent of the secant connecting the origin and the conditional point.

If SMA rods without pretraining or with inadequate pretraining are used in a self-centering system, considering that the number of loading cycles and the strain amplitude in each loading cycle would being experienced by the SMA rods during an earthquake event could not be predicted in advance, the behavior of the self-centering system will become unpredictable. In another aspect, since the forward transformation stress $\sigma_{\mathrm{Ms}}$ significantly decreases as the number of cycles increases, the linear strength of the self-centering system will decrease after an earthquake event. In another word, the strength of the self-centering system is not recovered and even the residual deformation of them is negligible. Whether the postearthquake recentered system with decreased strength could be used as before will be in question. This fact will contradict the original design philosophy of a self-centering system which is aimed to be reusable after designed earthquake without repairmen or with limited repairmen. Previous experimental studies also showed that there was an amount of unrecoverable deformation in the self-centering system caused by the incremental residual strain between the loading cycles of the SMA rods without pretraining [18]. The amount of this unrecoverable deformation is sometime very small and deemed negligible [18], but in some other scenarios, it might be deemed nonnegligible [12]. For the reasons mentioned above, this paper aims to obtain a kind of SMA rods with repeatable/predictable stress-strain loops and negligible residual strain through a proper pretraining.

To determine a proper pretraining scheme for the given SMA rods, four specimens with different heat treatment temperatures were firstly loaded with the loading protocol I and then loaded with the loading protocol II by 25 constant cycles, at a strain rate of $0.03 \% \mathrm{~s}^{-1}$, that is loaded with loading protocol LP (I-0.03 and II-0.03-25). The stress-strain responses of the specimens under the aforementioned loading protocol are shown in Figure 6. It is observed that most residual strain taken place at the first several cycles, and the accumulated residual strain becomes stable as the number of constant cycles reaches a certain value. Similar to the residual strain, most decrement of the forward transformation stress $\sigma_{\mathrm{Ms}}$ also happens in the first several cycles and approaches stable as the number of constant cycles 


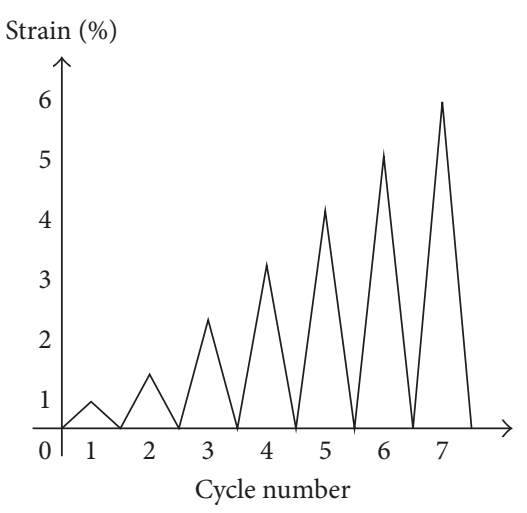

(a)

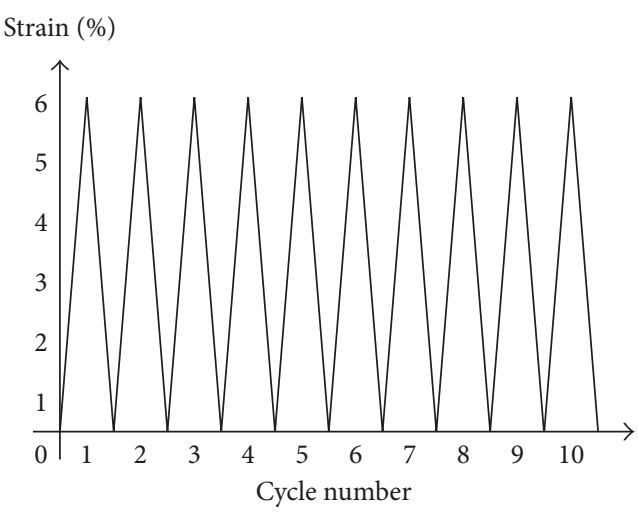

(b)

Figure 3: Two basic loading protocols. (a) LPI and (b) LPII.

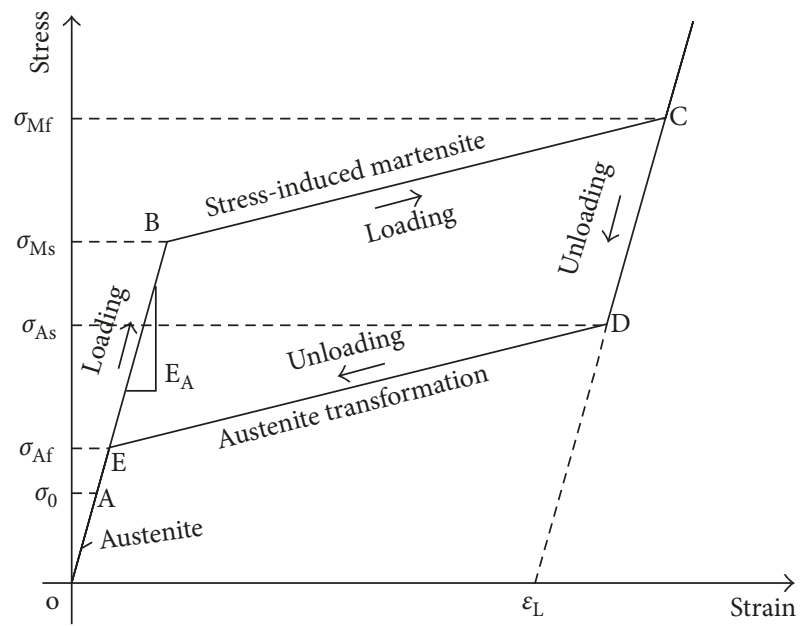

FIGURE 4: Diagram for the simplified stress-strain curve of superelastic SMA material.

increases. To quantify the development of the residual strain, the accumulated residual strain at typical load cycles are given in Table 2, where $\varepsilon_{0}, \varepsilon_{5}, \varepsilon_{10}, \varepsilon_{15}$, and $\varepsilon_{25}$ denote the accumulated residual strains after $0,5,10,15$, and 25 constant loading cycles, respectively. It shows that more than $80 \%$ accumulated residual strain had been taken place after 15 constant cycles for each specimen. A similar quantification on the forward transformation stress $\sigma_{\mathrm{Ms}}$ is given in Table 3. It shows that the decrement on the forward transformation stress was within $20 \%$ after 15 constant cycles. As a result, the incremental cycles and the 15 constant cycles loaded at a strain rate of $0.03 \% \mathrm{~s}^{-1}$ are chosen as the prestraining loading scheme for the SMA specimens.

\subsection{Effect of Heat Strategy}

3.2.1. Heat Temperature. As mentioned in Section 2, to investigate the effect of heat strategy on the mechanical properties, 4 heat temperatures evenly ranged from $300^{\circ} \mathrm{C}$ to $450^{\circ} \mathrm{C}$ with same duration of 30 minutes and 2 heat sequences: heat before machining and heat after machining were considered.

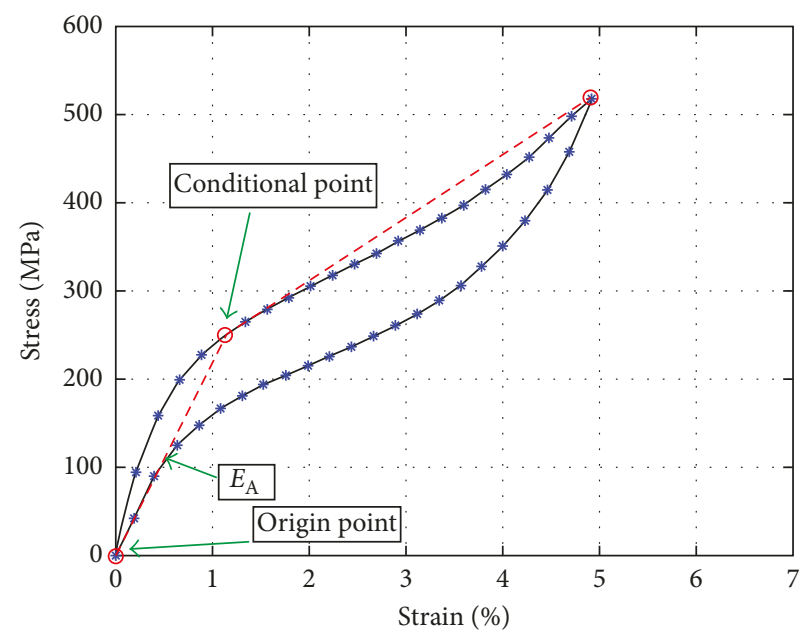

FIGURE 5: Diagram for the stress-strain response of real SMA material.

A specimen without heat treatment and four specimens annealed with different temperatures ranged from $300^{\circ} \mathrm{C}$ to $450^{\circ} \mathrm{C}$ were tested using a same loading protocol as that used in Section 2. Note that the mechanical properties of the SMA rods after the pretraining are interested by this paper. Thus, the average mechanical properties exhibited in the last ten loading cycles of the specimens were used to represent the mechanical properties of the specimens after pretraining. The detailed results on the mechanical properties of the specimens after pretraining are plotted in Figure 7. It is clearly shown that both the residual strain and the elastic modulus of the specimens firstly decrease as the heat temperature increases and then increase as the heat temperature increases. The residual strain reaches the minimum $1.15 \%$ at $350^{\circ} \mathrm{C}$, and the elastic modulus reaches the minimum $15 \mathrm{GPa}$ at $300^{\circ} \mathrm{C}$. The equivalent viscous damping (EVD) of the specimens firstly increases as the heat temperature increases and then decreases as the heat temperature increases. It reaches the maximum $2.22 \%$ at $400^{\circ} \mathrm{C}$. It is also worth noting that the maximum EVD at $400^{\circ} \mathrm{C}$ is lower than the typical value required for the purpose of major 


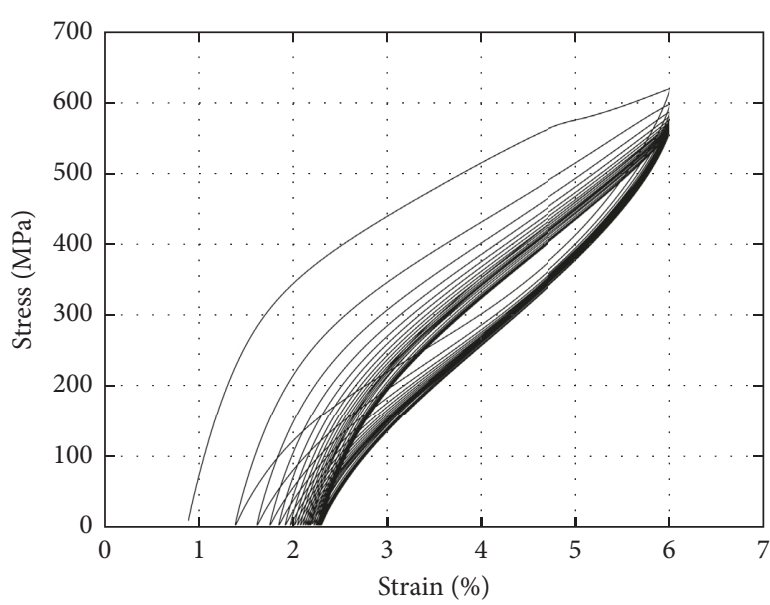

- 14-300-30-MH

(a)

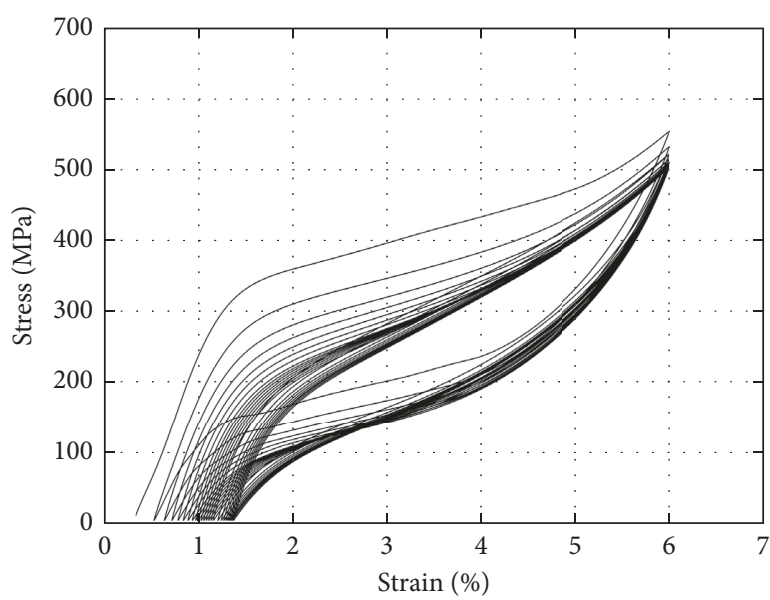

- 14-400-30-MH

(c)

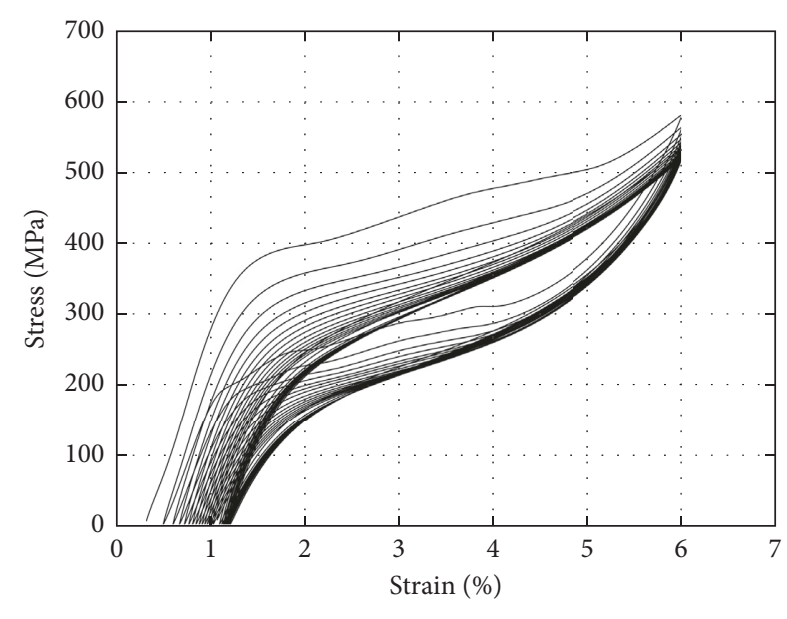

- 14-350-30-MH

(b)

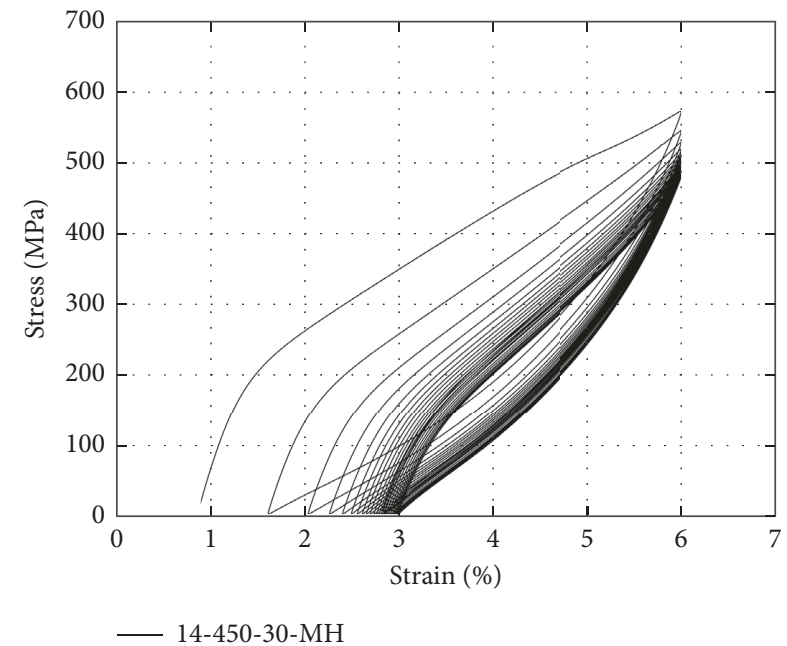

(d)

FIgURE 6: Stress-strain responses under loading protocol LP (I-0.03 and II-0.03-25).

TABle 2: Accumulated residual strains at typical loading cycles.

\begin{tabular}{|c|c|c|c|c|c|c|c|c|c|c|c|}
\hline \multirow{3}{*}{ Specimens } & \multicolumn{11}{|c|}{ Accumulated residual strains } \\
\hline & \multicolumn{2}{|c|}{$\begin{array}{l}\text { After incremental } \\
\text { cycles }\end{array}$} & \multicolumn{2}{|c|}{$\begin{array}{c}\text { After } 5 \text { constant } \\
\text { cycles }\end{array}$} & \multicolumn{2}{|c|}{$\begin{array}{l}\text { After } 10 \text { constant } \\
\text { cycles }\end{array}$} & \multicolumn{2}{|c|}{$\begin{array}{l}\text { After } 15 \text { constant } \\
\text { cycles }\end{array}$} & \multicolumn{2}{|c|}{$\begin{array}{c}\text { After } 20 \text { constant } \\
\text { cycles }\end{array}$} & \multirow{2}{*}{$\begin{array}{c}\text { After } 25 \text { constant } \\
\text { cycles } \\
\varepsilon_{25}(\%) \\
\end{array}$} \\
\hline & $\varepsilon_{0}(\%)$ & $\varepsilon_{0} / \varepsilon_{25}(\%)$ & $\varepsilon_{5}(\%)$ & $\varepsilon_{5} / \varepsilon_{25}(\%)$ & $\varepsilon_{10}(\%)$ & $\varepsilon_{10} / \varepsilon_{25}(\%)$ & $\varepsilon_{15}(\%)$ & $\varepsilon_{15} / \varepsilon_{25}(\%)$ & $\varepsilon_{20}(\%)$ & $\varepsilon_{20} / \varepsilon_{25}(\%)$ & \\
\hline D14/14-N-N & 3.56 & 89.67 & 3.87 & 97.48 & 3.93 & & 3.95 & 99.50 & 3.96 & 99.75 & 3.97 \\
\hline D14/14-300-30 & 1.38 & 60.00 & 1.91 & 83.04 & 2.10 & 91.30 & 2.20 & 95.65 & 2.27 & 98.70 & 2.30 \\
\hline D14/14-350-30 & 0.49 & 40.50 & 0.76 & 62.81 & 0.93 & 76.86 & 1.06 & 87.60 & 1.16 & 95.87 & 1.21 \\
\hline D14/14-400-30 & 0.53 & 38.13 & 0.84 & 60.43 & 1.03 & 74.10 & 1.16 & 83.45 & 1.31 & 94.24 & 1.39 \\
\hline D14/14-450-30 & 1.61 & 53.67 & 2.49 & 83.00 & 2.73 & 91.00 & 2.86 & 95.33 & 2.93 & 97.67 & 3.00 \\
\hline
\end{tabular}

damping. The EVD at $350^{\circ} \mathrm{C}$ is $1.72 \%$ which is lower than that at $400^{\circ} \mathrm{C}$ but higher than the others. The forward transformation stress always decreases as the heat temperature increases. For the purposed application, priority is given to the self-centering ability. Hence, $350^{\circ} \mathrm{C}$ resulting minimum residual strain is chosen as the optimal heat temperature.

Note that the effects of heat temperature (duration $=30$ mins) on the mechanical properties of the SMA rods found in this paper differ from those reported by Wang et al. [18]. It is believed that the main reason for the differences is that the specimens of SMA rods used here have been pretrained.

3.2.2. Heat and Machining Sequence. As reported by Wang et al. [18], when the $20 \mathrm{~mm}$ diameter SMA rods were heated firstly and then machined to the dog-bone shape, the optimal 
TABLE 3: Forward transformation stress at typical loading cycles.

\begin{tabular}{lccccccccccc}
\hline & \multicolumn{1}{c}{$\begin{array}{c}\text { Forward transformation stress } \\
\text { Specimens }\end{array}$} & $\begin{array}{c}\text { After } \\
\text { incremental } \\
\text { cycles }\end{array}$ & $\begin{array}{c}\text { After } 5 \text { constant } \\
\text { cycles }\end{array}$ & $\begin{array}{c}\text { After } 10 \text { constant } \\
\text { cycles }\end{array}$ & $\begin{array}{c}\text { After } 15 \text { constant } \\
\text { cycles }\end{array}$ & $\begin{array}{c}\text { After } 20 \text { constant } \\
\text { cycles }\end{array}$ & $\begin{array}{c}\text { After } 25 \text { constant } \\
\text { cycles }\end{array}$ \\
& $\sigma_{0}(\mathrm{MPa})$ & $\sigma_{0} / \sigma_{25}$ & $\sigma_{5}(\mathrm{MPa})$ & $\sigma_{5} / \sigma_{25}$ & $\sigma_{10}(\mathrm{MPa})$ & $\sigma_{10} / \sigma_{25}$ & $\sigma_{10}(\mathrm{MPa})$ & $\sigma_{10} / \sigma_{25}$ & $\sigma_{10}(\mathrm{MPa})$ & $\sigma_{10} / \sigma_{25}$ & $\sigma_{10}(\mathrm{MPa})$ \\
\hline $\mathrm{D} 14 / 14-\mathrm{N}-\mathrm{N}$ & 674 & 1.06 & 657 & 1.04 & 650 & 1.03 & 644 & 1.02 & 640 & 1.01 & 633 \\
$\mathrm{D} 14 / 14-300-30$ & 605 & 1.10 & 578 & 1.05 & 568 & 1.04 & 557 & 1.02 & 551 & 1.01 \\
$\mathrm{D} 14 / 14-350-30$ & 399 & 1.65 & 313 & 1.29 & 275 & 1.14 & 258 & 1.07 & 244 & 1.01 \\
$\mathrm{D} 14 / 14-400-30$ & 360 & 1.95 & 253 & 1.40 & 216 & 1.19 & 202 & 1.12 & 188 & 1.04 \\
$\mathrm{D} 14 / 14-450-30$ & 210 & 1.44 & 175 & 1.20 & 163 & 1.12 & 155 & 1.06 & 150 & 1.03 \\
\hline
\end{tabular}

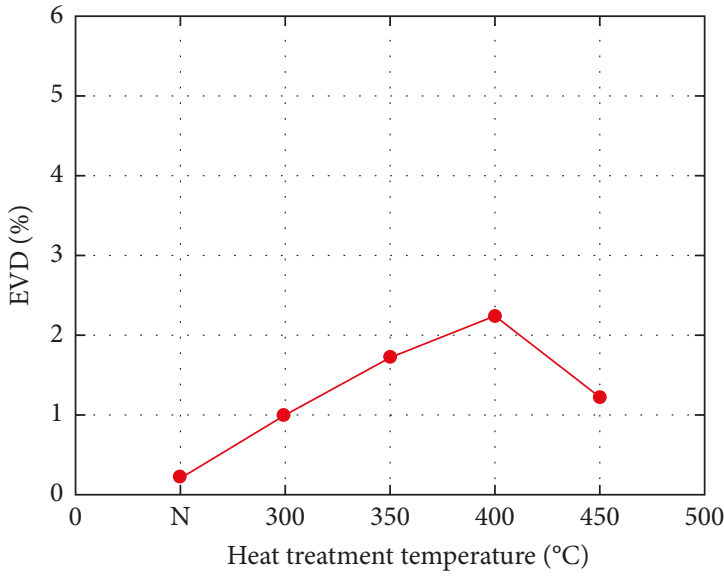

(a)

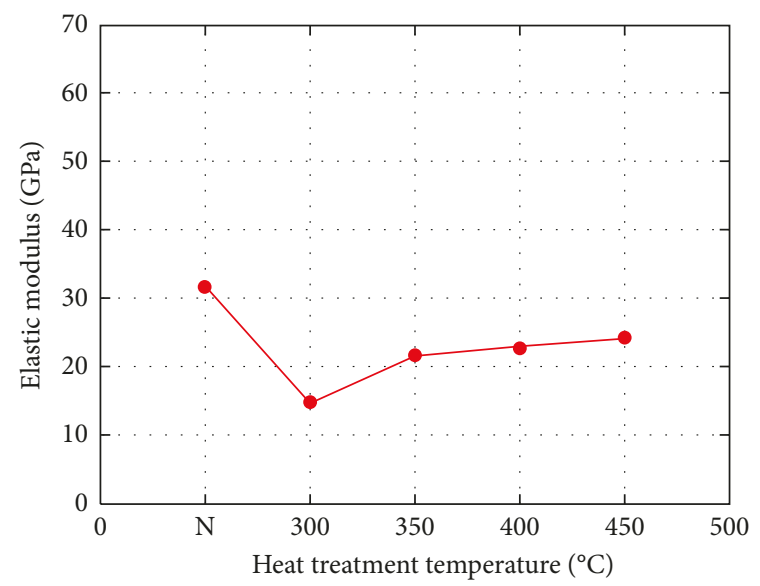

(c)

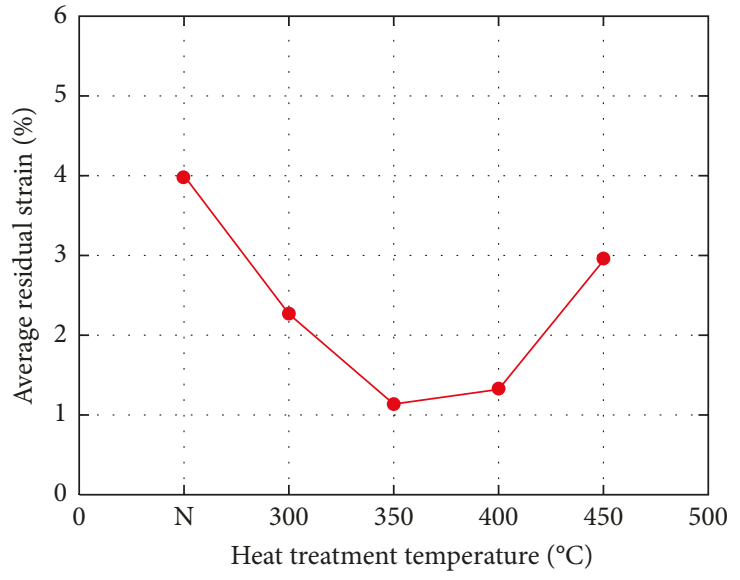

(b)

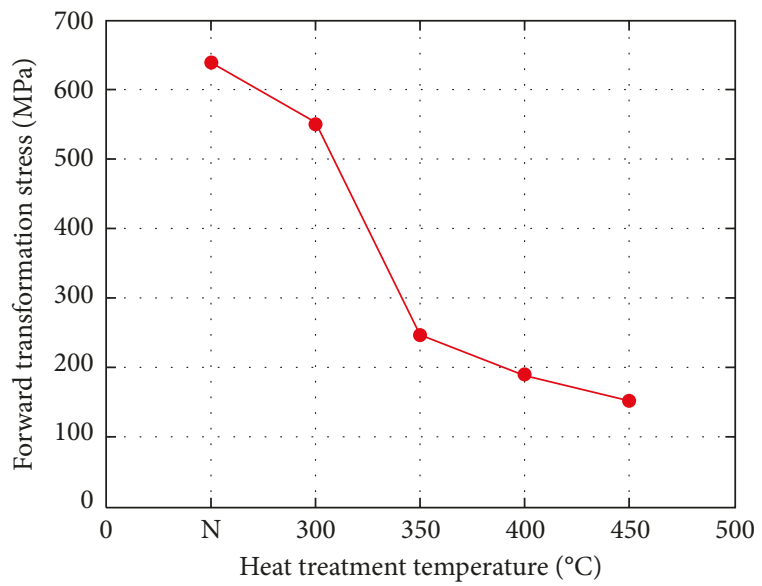

(d)

FIGURE 7: Effect of heat temperatures on the mechanical properties. Effect on (a) EVD, (b) average residual strain, (c) elastic modulus, and (d) forward transformation stress.

heat temperature was $450^{\circ} \mathrm{C}$ with the duration of 30 minutes. Since the material used in this paper was purchased from the same manufacture as that used by Wang el al. [18] and had a similar content to that used by Wang el al. [18], the optimal heat strategy proposed by the previous study was supposed to be directly adopted by this paper. But it was found that the SMA rods became harder after heating treatment and thus were more expensive for the machining. As a result, machining before heating treatment was finally adopted by this study. To check the effect of the sequence exchanging between machining and heating treatment on the mechanical properties of the SMA rods, comparison tests on a specimen using the previous heating-machining pretreatment (i.e., specimen 14-45030-HM) and a specimen using machining-heating pretreatment (i.e., specimen 14-450-30-MH) were conducted. The previously proposed optimal heating strategy (i.e., $450^{\circ} \mathrm{C}$ with the duration of 30 minutes) and the loading protocol LP 


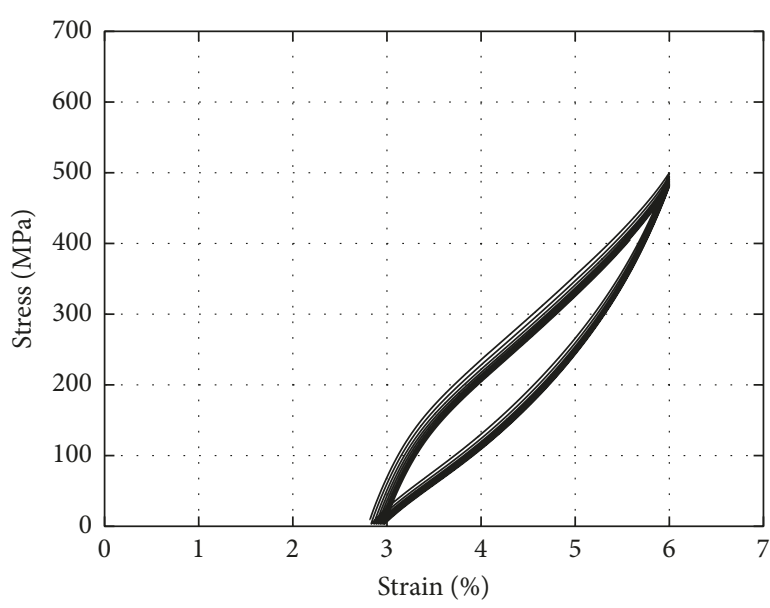

(a)

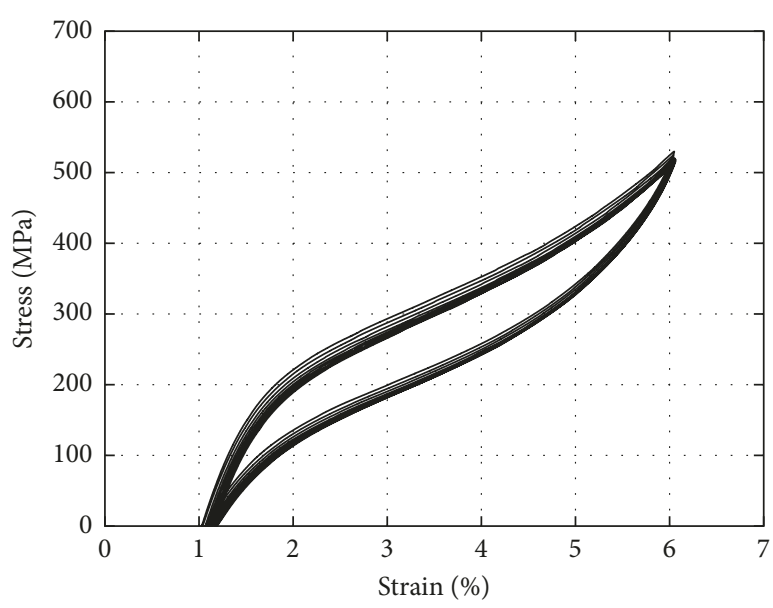

(b)

Figure 8: Cyclic responses of specimens using different pretreatment sequences. Cyclic responses of specimen (a) 14-450-30-MH and (b) 14-450-30-HM.

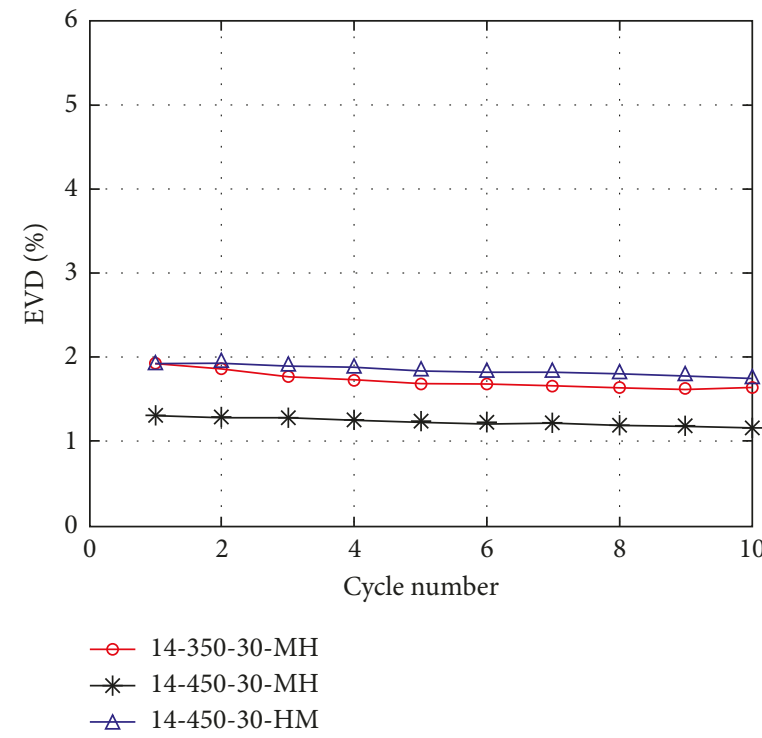

(a)

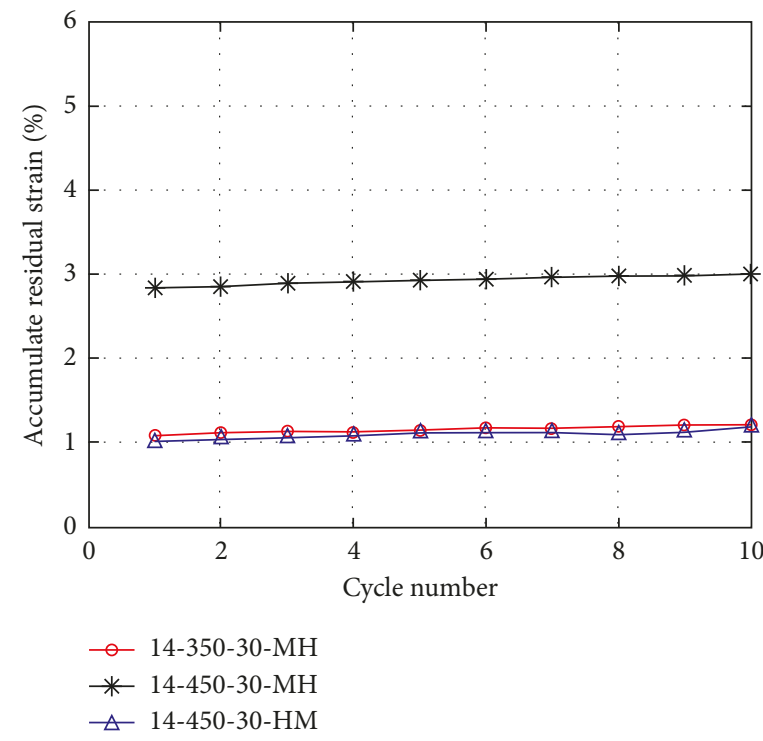

(b)

Figure 9: Effect of heat and machining sequence on the mechanical properties. Comparison on (a) EVD and (b) accumulated residual strain.

(I-0.03 and II-0.03-25) were used for both specimens. The after pretraining cyclic responses of the two specimens are given in Figure 8.

It is found that the specimen 14-450-30-HM exhibits a comparable response to its counterpart reported in the previous study [18]. The specimen 14-450-30-MH exhibits poor performance in both self-centering capacity and energy dissipation capacity, compared to the specimen 14-450-30-HM, as shown in Figure 9. It is also shown that the specimen 14-35030-MH exhibits comparable performance to the specimen 14450-30-HM. These observations indicate that optimal heating strategy determined by the $20 \mathrm{~mm}$ diameter raw SMA rods is not applicable for the machined $14 \mathrm{~mm}$ diameter specimen. In another words, the optimal heating strategy is size dependent. As found in Section 3, the optimal heating strategy determined for the machined $14 \mathrm{~mm}$ diameter SMA specimens is $350^{\circ} \mathrm{C}$ with the duration of 30 minutes.

3.3. Effect of Strain Rate. To investigate the effect of strain rates on the mechanical properties of the SMA rods, three identical specimens $14-350-30-\mathrm{MH}$ are pretrained at $0.03 \%$ strain rate and then loaded 10 constant cycles at $0.03 \%$, $0.15 \%$, and $0.30 \%$ strain rates, respectively. The cyclic responses of them are shown in Figure 10. It can be observed that the three pretrained specimens loaded at different strain rates exhibit similar responses with negligible residual strain. The area enveloped by the stress-strain cycles is seemed to get smaller as the strain rate increases. This observation has been verified by the plots on the average EVD and the 


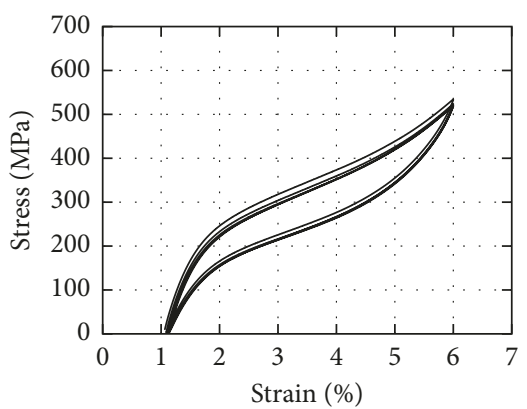

(a)

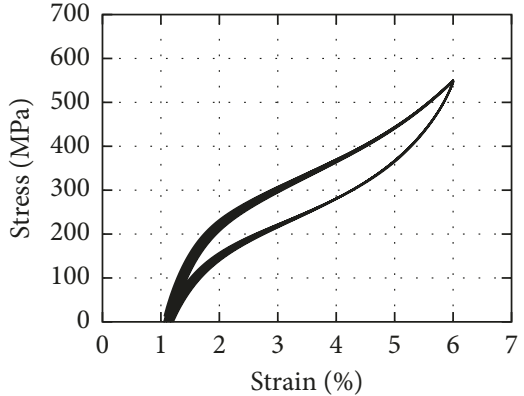

(b)

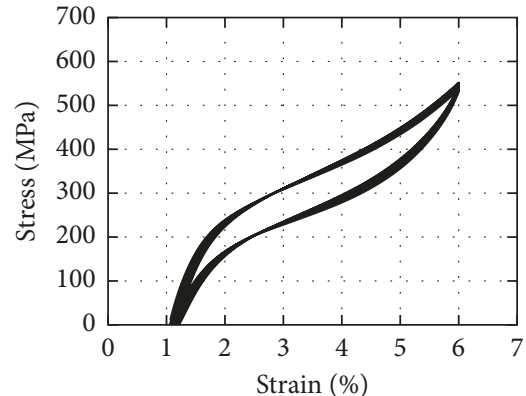

(c)

FIGURE 10: Stress-strain responses under different loading rates. Stress-strain response under loading rate (a) $0.03 \%$, (b) $0.15 \%$, and (c) $0.3 \%$.

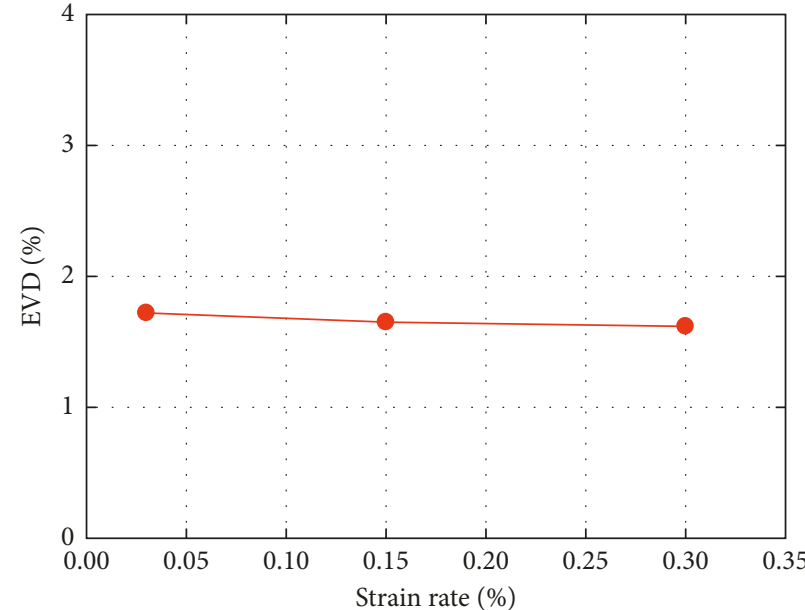

(a)

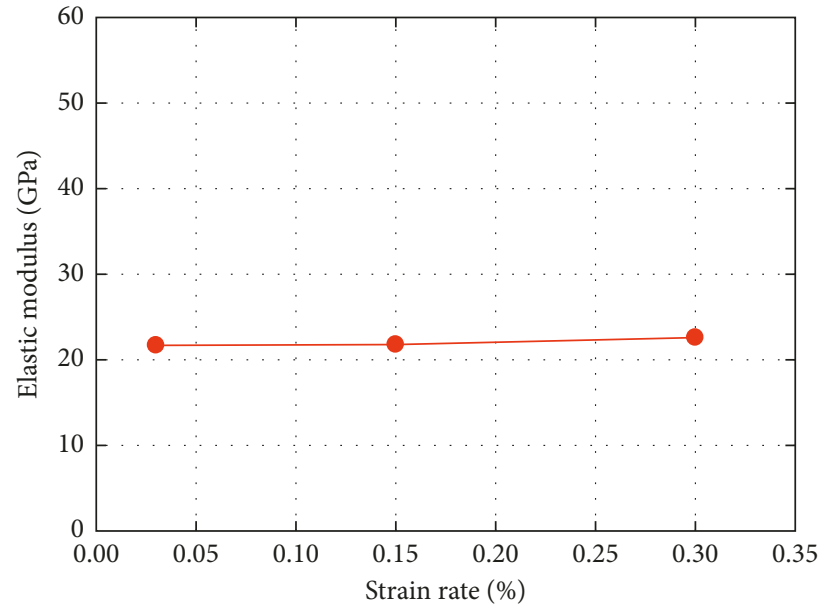

(c)

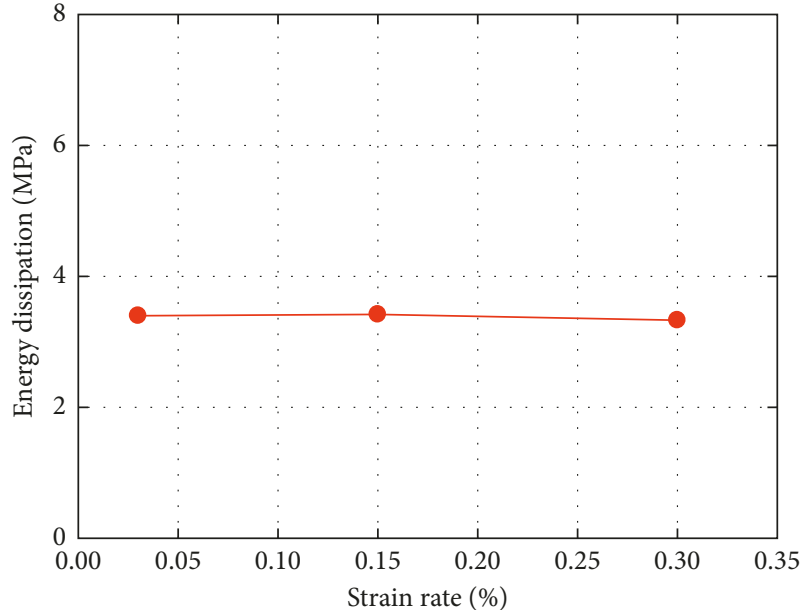

(b)

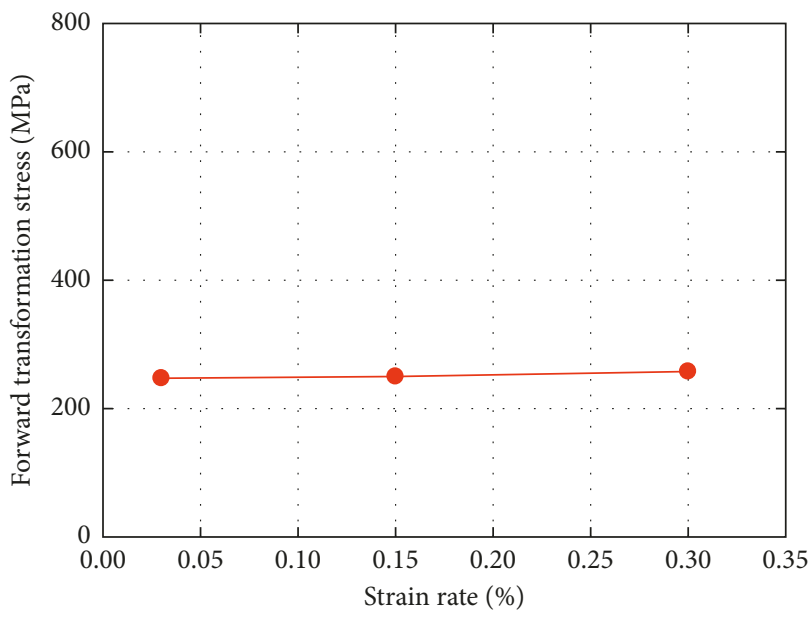

(d)

FIGURE 11: Effect of loading rate on mechanical properties. Effect on (a) EVD, (b) energy dissipation, (c) elastic modulus, and (d) forward transformation stress.

average energy dissipation of the 10 cycles for each specimens (Figures 11(a) and 11(b)). It is also found that the average elastic modulus and the average forward transformation stress slightly increase as the strain rate increases (Figures 11(c) and 11(d)). This effect can be explained by the observation that the specimen loaded at a higher strain rate got hotter than the counterpart loaded at a lower strain rate. The heat released during the martensite transformation cannot be dissipated at higher strain rate and thus causes the temperature of the specimen increasing. The increasing of the specimen temperature causes the forward transformation stress increasing and then results a larger elastic modulus. 
3.4. Effect of Strain Amplitude. A specimen 14-350-30-MH is loaded by the protocol LP (I-0.03, II-0.03-15 and I-0.03) to investigate the effect of strain amplitude on the mechanical properties of the prestrained SMA rods. The stress-strain response of the specimen under different strain amplitudes is shown in Figure 12, and the corresponding changes in mechanical properties are shown in Figure 13. It is found that the dissipated energy increases as the strain amplitude increases and the corresponding EVD increases as the strain amplitude increases from. Therefore, to fully exploit the energy dissipation capacity of the material, the self-centering system should be properly designed to ensure that the SMA rods undergo high strain amplitude in the target earthquake scenario.

Since the strain plateau has not fully developed in the cycles with strain amplitudes smaller than $4 \%$, there is a skip in the elastic modulus and the forward transformation stress between the cycle with $4 \%$ strain and the cycle with $5 \%$ strain. For the cycles above 5\% strain, both the elastic modulus and the forward transformation stress gradually decrease as the strain amplitude increases. This may not be purely caused by the increasing of the strain amplitude. There may be also contribution from the increasing of the number of cycles as that observed in the constant loading cycles.

\section{Simplified Material Model for FEM Analysis}

4.1. Simplified Material Model. The optimal pretreatment determined by the tests will be adopted for the SMA rods that will be applied in self-centering link beams. FE-based numerical simulation is a well-accepted method to predict the response or parametric study of the performance of a structural system/component before or after test study. Aurichhio's model $[19,20]$ is usually employed by commercial FE packages (e.g., ANSYS and ABAQUS) to model the SE effect of nitinol SMA at isothermal condition. To numerically analyze the self-centering link beams using the above tested SMA rods, the stress-strain response obtained from tests is needed to be simplified into Aurichhio's model. In this paper, the commercial FE package ANSYS was intended to be employed to carry out the numerical study. ANSYS uses 6 parameters including transformation stresses $\left(\sigma_{\mathrm{Ms}}, \sigma_{\mathrm{Mf}}, \sigma_{\mathrm{As}}\right.$, and $\left.\sigma_{\mathrm{Af}}\right)$, Young's modulus $\left(E_{\mathrm{A}}\right)$, and the maximum transformation strain $\left(\varepsilon_{\mathrm{L}}\right)$ to define Aurichhio's model for the SE SMA. A typical six-parameter model for SMA material is shown in Figure 14. It clearly shows that the material model is defined as long as the five points $\mathrm{O}$ and A-D are determined.

As mentioned in Section 3, the point A is defined as the point with maximum curvature on the loading curve. The origin $\mathrm{O}$ is defined as the starting point of the loading cycle. The point with maximum recoverable strain is defined as point $\mathrm{B}$. The characteristic points $\mathrm{C}$ and $\mathrm{D}$ are defined as follows. Similar to the definition of point $\mathrm{A}$, the point $\mathrm{E}$ with maximum curvature on the unloading curve is kept on the simplified model. As a result, the line CD must pass through the point E. Meanwhile, it is assumed that line $\mathrm{BC}$ is parallel to line $\mathrm{OA}$ and further required that the envelope of the simplified stress-strain curve is equal to the envelope of the

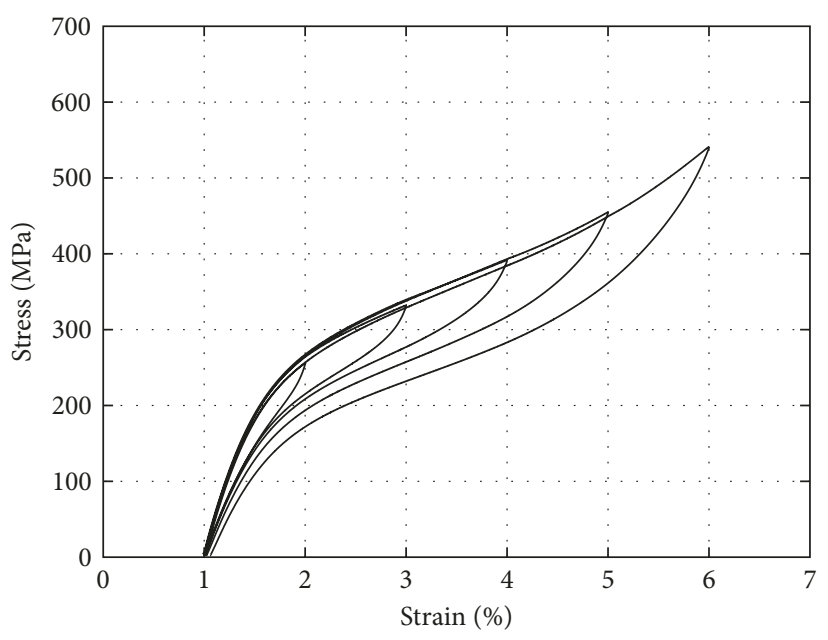

FIGURE 12: Stress-strain responses under different strain amplitudes.

experimental stress-strain curve. Under the above conditions, the locations of points $\mathrm{C}$ and $\mathrm{D}$ can be determined.

The simplified model for the pretrained specimen 14$350-30-\mathrm{MH}$ is determined using the above principle, and the corresponding characteristic parameters are given in Table 4 .

Note that a few stress-strain models for SMA rods have been proposed and used in the previous numerical studies on SMA-based self-centering systems $[11,14,21,22]$. But, there are two debatable details as follows in those previous models:

(1) Most of them were based on the first-cycle test data of SMA rods without prestraining. As we know, the SMA rods without prestraining are not able to repeat the response of its first cycle in the following loading cycles, while multicycle loading is unavoidable in a real earthquake event. There would be a considerable error between the numerically predicted response and the real response after the first cycle.

(2) There is no clear equivalent principle having being stated for the derivation from the test data to the simplified model. As a result, different simplified models might be derived from the same test data.

\subsection{FE Simulation on Self-Centering Link Beam Using SMA Rods}

4.2.1. Prototype System. Using the simplified SMA material model obtained in this paper and the analytical model for self-centering link beams using SMA rods given by Xu et al. [14], a $950 \mathrm{~mm}$ self-centering link beam with a shear strength demand of $130 \mathrm{kN}$ and an ultimate rotation demand of $0.08 \mathrm{rad}$ was designed. Note that the $0.08 \mathrm{rad}$ link rotation demand was appointed in accordance with the requirement specified by AISC (2010) for a shear yield link [23]. An overall diagram for the self-centering link beam is shown in Figure 15(a). A prestress $\sigma_{0}=\sigma_{\mathrm{Af}}$ is used to get a high linear elastic strength. Grade Q345 hot rolled H-shape steel HM300 $\times 200$ [24] is used for the link beam. At each end of 


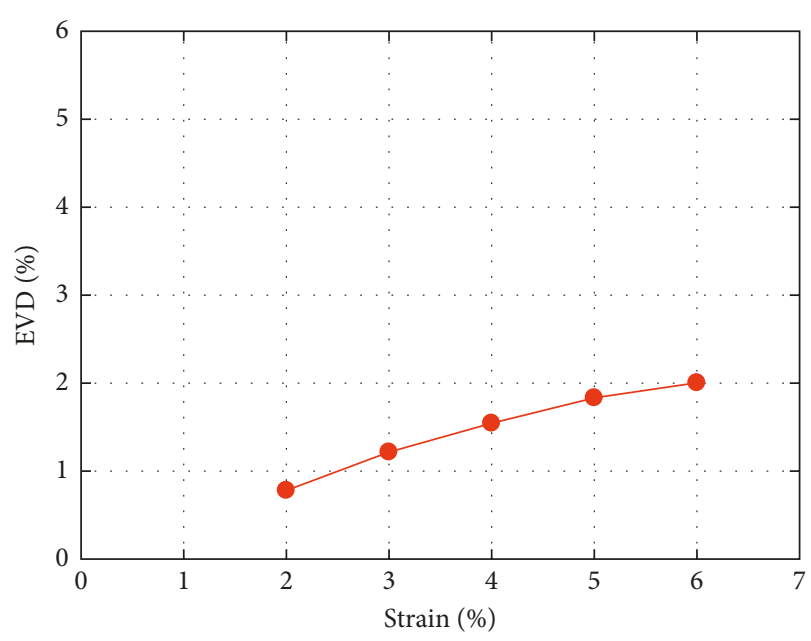

(a)

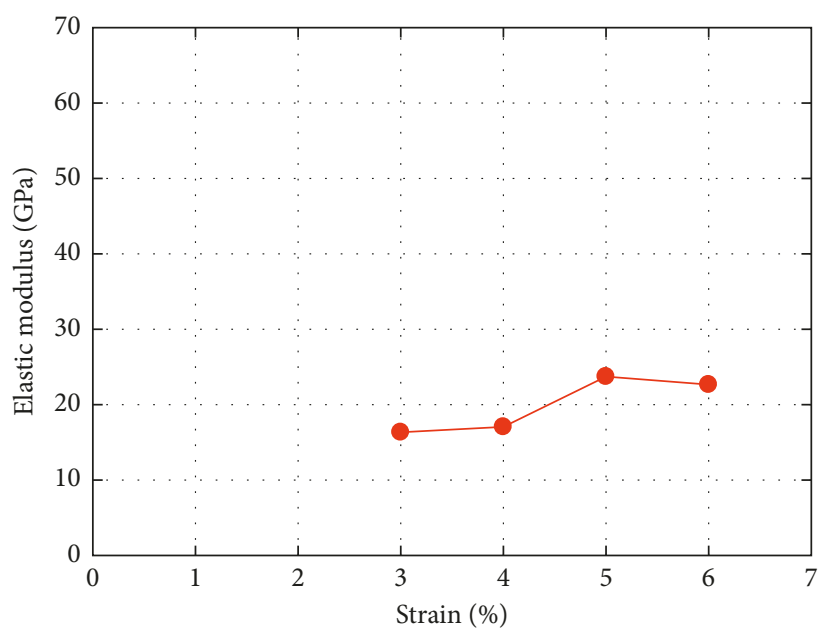

(c)

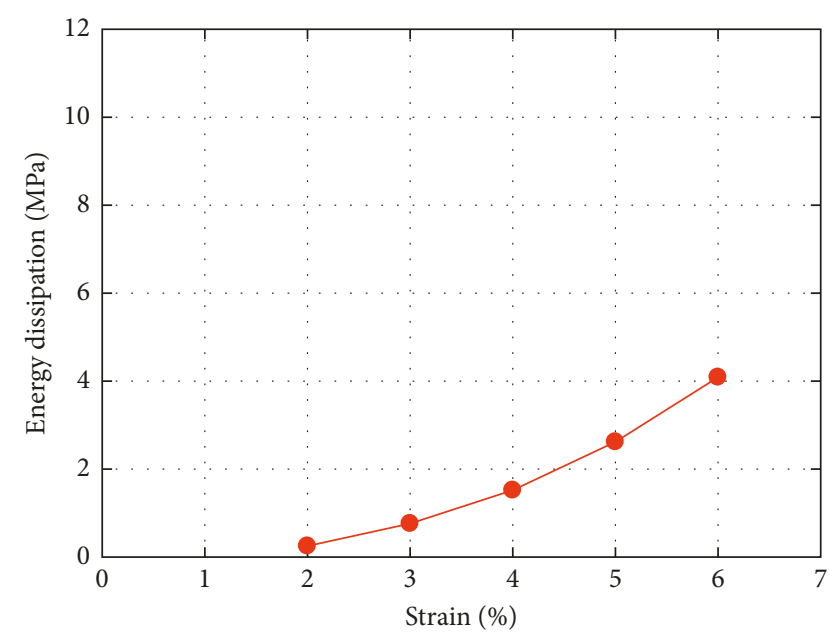

(b)

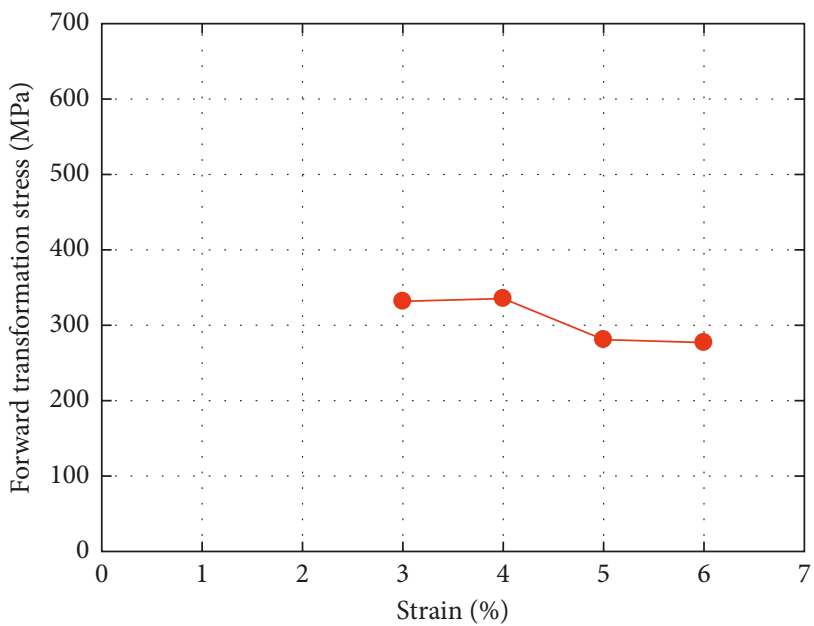

(d)

FIGURE 13: Effect of strain amplitude on mechanical properties. Effect on (a) EVD, (b) energy dissipation, (c) elastic modulus, and (d) forward transformation stress.

the link, the outer $300 \mathrm{~mm}$ flanges are thickened to $27 \mathrm{~mm}$ to stiffen the end corners of the link; otherwise, there will be plastic deformation intensively developed in the flange and web around the link end corner which acts as the pivot during the gap opening [21]. Young's modulus of $206 \mathrm{GPa}$ and a yield strength of $345 \mathrm{MPa}$ are assumed for steel material. 6 SMA rods with a length of $510 \mathrm{~mm}$ and a net diameter of $14 \mathrm{~mm}$ are used for each end connection of the link beam to achieve the demanded performance. They are fixed to the anchor plates. Stiffeners are added to the anchor plates to enhance their bending stiffness. The layout of the SMA rods on the end plate is shown in Figure 15(b).

A virtual test setup which is similar to the real test setup used by Mansour et al. [25, 26], as shown in Figure 16, is used in the simulation. A cross section of $500 \mathrm{~mm}$ (depth) $\times$ $200 \mathrm{~mm}$ (flange width) $\times 10 \mathrm{~mm}$ (web thickness) $\times 16 \mathrm{~mm}$ (flange thickness) is assumed for the test beams. Grade Q345 steel is also used for the test beams. The loading beams are assumed to be rigid enough that its deformation can be neglected.

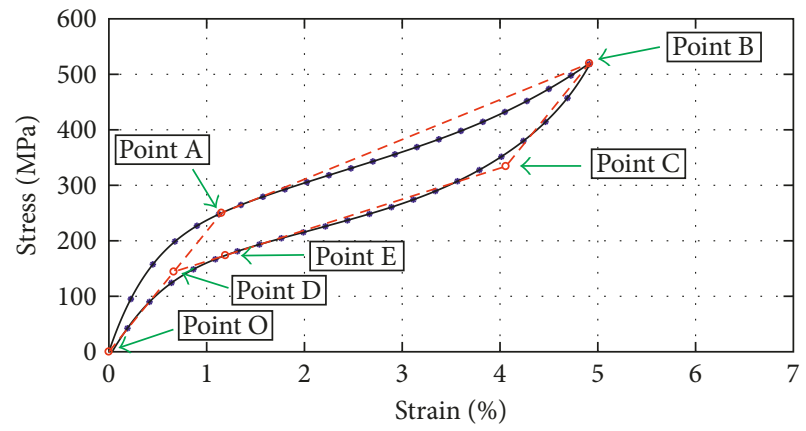

FIGURE 14: Derivation of simplified SMA material model from test data.

4.2.2. FE Model. Since the whole structural system is symmetry about the middle-thickness plane of the web of the link beam, only half of the structural system is modeled in the simulation. Solid elements were used for the SMA rods, the link beam, and the test beams. Line elements were used 
TABLE 4: Characteristic parameters of simplified model for specimen 14-350-30-MH.

\begin{tabular}{lcccccc}
\hline Parameters & $E_{\mathrm{A}}(\mathrm{GPa})$ & $\sigma_{\mathrm{Ms}}(\mathrm{MPa})$ & $\sigma_{\mathrm{Mf}}(\mathrm{MPa})$ & $\sigma_{\mathrm{As}}(\mathrm{MPa})$ & $\sigma_{\mathrm{Af}}(\mathrm{MPa})$ & $\varepsilon_{\mathrm{L}}$ \\
\hline Values & 21.7 & 254.6 & 524.2 & 338.7 & 148.7 & 0.025 \\
\hline
\end{tabular}

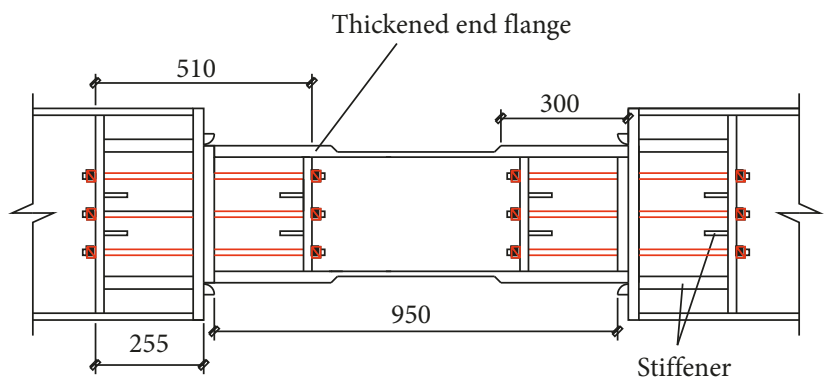

(a)

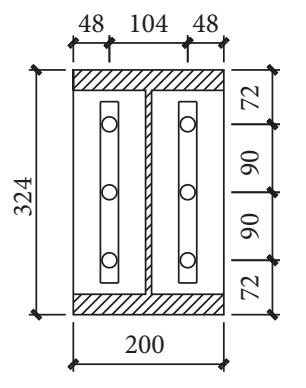

(b)

FIGURE 15: Prototype self-centering link beam considered in FE simulation: (a) self-centering link beam and (b) layout of SMA rods.

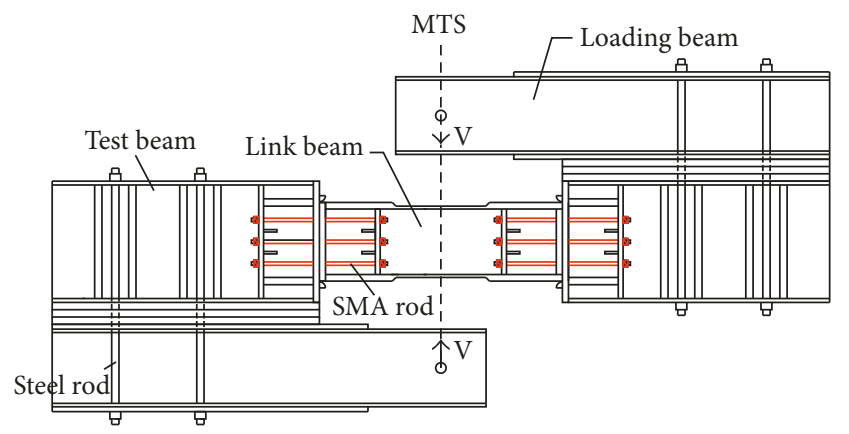

Figure 16: Virtual test setup.

for the loading beams to reduce the number of elements and enhance the computing efficiency, as shown in Figure 17.

The general-purpose FE software package ANSYS v.12.1 [27] was used to conduct the simulation. 3D solid element SOLID185 with eight nodes was employed for the SMA rods, the link beam, and test beams. They were meshed by mapped mesh except the shear keys. 3D 2-node beam element BEAM188 was used to model the rigid loading beams. Multipoint constraint element MPC184 was used to connect loading beams with test beams. The contact pair CONTA173 and TARGE170 was used to simulate the physical contact between the link beam and the test beams, as well as the contact between the steel nuts and anchor plates. "Hard contact" behavior with no penetration in the normal direction was assumed for all the contact pairs. A friction coefficient of 0.3 was used for all the contact surfaces. The pretension in the SMA rods was introduced by creating two pretension sections PRETS179 at the middle of each rod and then applying the given pretension onto the sections. The whole established FE model of the structural system is shown in Figure 17.

The steel material was simulated by the bilinear kinematic hardening model built in ANSYS. Young's modulus and yield strength mentioned above had been used in the

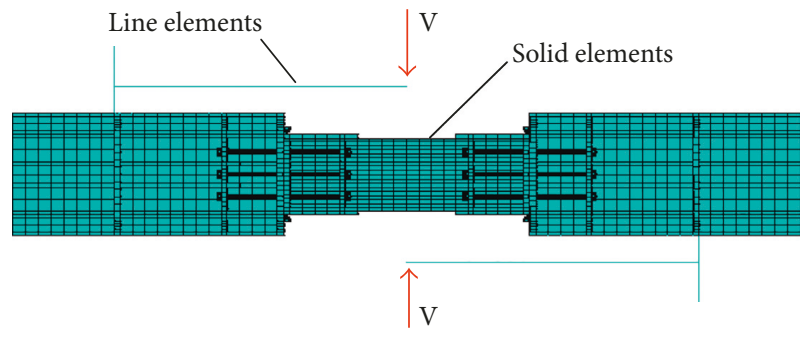

FIGURE 17: FE model of self-centering steel link beam.

simulation. The strain hardening modulus of the steel material was assumed to be $15 \mathrm{GPa}$. The six-parameter SMA model determined above was simulated by ANSYS's built-in superelastic SMA model.

A loading protocol which cyclically loaded the link to a rotation of $0.02 \mathrm{rad}, 0.04 \mathrm{rad}, 0.06 \mathrm{rad}$, and $0.08 \mathrm{rad}$ was employed.

4.2.3. Result of Numerical Simulation. The deformation as well as the distribution of the Von Mises stress of the structural system at the ultimate state $(0.08 \mathrm{rad})$ is shown in Figure 18. It is observed that the stress of all the steel components keeps below the yield strength. The shear force to link the rotation curve obtained from the simulation exhibits a "flag-like" shape, and there is no global residual deformation (Figure 19). The stress-strain response of a typical SMA rod located in the outer rows is shown in Figure 20. It is shown that, as expected, it behaves in a manner in accordance with the simplified material model.

\section{Conclusions}

This paper has conducted a number of tests on pretrained large size SMA rods which are towards to be used in a new concept self-centering steel link beam. The pretraining scheme for the given SMA rods is determined based on the test results. The effects of quenching temperature, strain rate, 


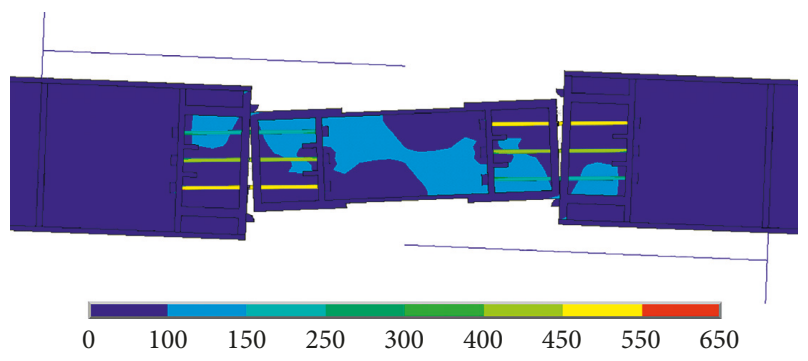

Figure 18: Deformation and stress distribution at ultimate state (unit: $\mathrm{MPa}$ ).

strain amplitude, and processing sequence on the mechanical properties of pretrained SMA rods are investigated. The optimal quenching temperature and the proper processing sequence are determined. A simplified stress-strain model for the prestrained SMA rods oriented to apply in numerical simulations is obtained based on the test results. An example of using the simplified SMA material model in numerical analysis of a self-centering steel link beam is conducted to validate the applicability of the material model. More detailed conclusions of this study are given as follows:

(1) The stress-strain response of the SMA rod under cyclic load significantly varies at the first a few cycles and then becomes stable after a certain number of cycles. Based on the test results, a pretraining scheme that consists of 6 incremental cycles and 15 constant cycles is determined.

(2) The quenching temperature shows a significant effect on mechanical properties of the pretrained SMA rod specimens. By comprehensively investigating the effect of it on the residual strain, elastic modulus, energy dissipation, and forward transformation stress, $350^{\circ} \mathrm{C}$ (duration $=30$ minutes) is deemed as the optimal quenching temperature.

(3) Shifting the order of machining process and heat treatment results in significant degeneration on the mechanical properties of the SMA rod specimen. This is because the optimal quenching temperature for the SMA rod specimen is sensitive to the effective diameter of the SMA rod specimen at the heat treatment. Machining before heat treatment is preferred for ease of machining.

(4) When the loading rate increases, the energy dissipation and the EVD decrease and the elastic modulus and forward transformation stress increase. Generally speaking, the decrements in energy dissipation and EVD and the increments in the elastic modulus and forward transformation stress are insignificant and may be negligible in most cases.

(5) When the strain amplitude increases, the energy dissipation and EVD linearly increase. This indicates that the SMA rod should be designed to be loaded into high-strain levels to fully extract the energy dissipation capacity of it.

(6) The applicability of the simplified stress-strain model for the pretrained SMA rods is verified by a numerical

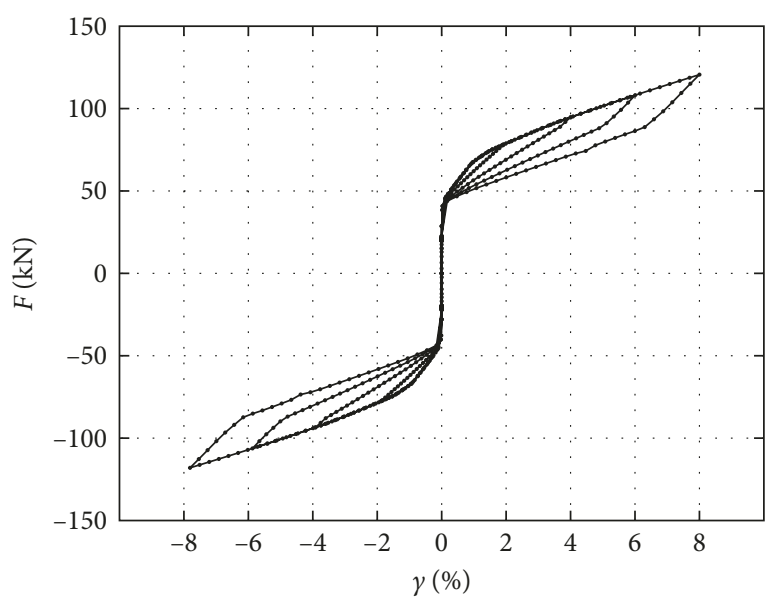

FIGURE 19: Shear force to link rotation response.

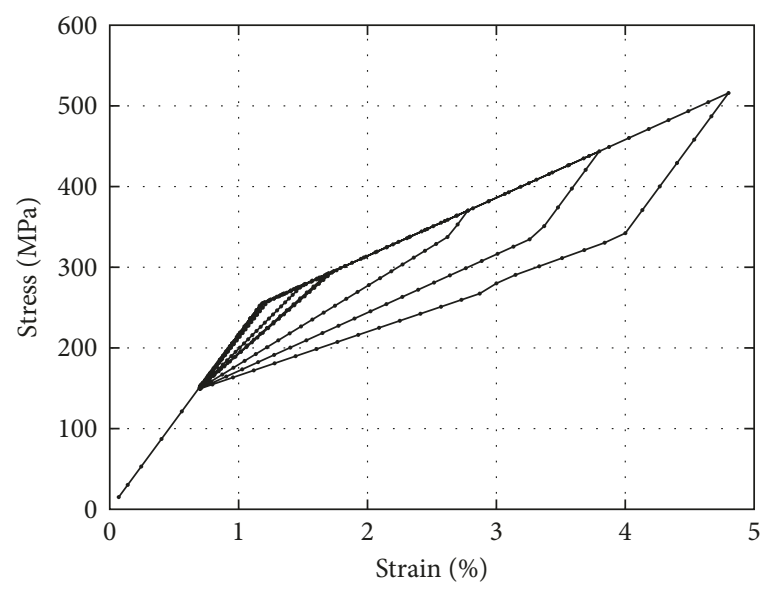

FIgURE 20: Stress-strain response of a typical SMA rod.

example. The numerical example shows that the prestrained SMA rods enable the self-centering link beam to be totally recoverable from a deformation up to $8 \%$ rotation.

\section{Conflicts of Interest}

The authors declare that there are no conflicts of interest regarding the publication of this manuscript.

\section{Acknowledgments}

This work is supported by the National Key Research and Development Program of China (Grant no. 2016YFC0800206) and the Fundamental Research Funds for the Central Universities.

\section{References}

[1] E. J. Graesser and F. A. Cozzarelli, "Shape-memory alloys as new materials for aseismic isolation," Journal of Engineering Mechanics, vol. 117, no. 11, pp. 2590-2608, 1991. 
[2] A. S. Whittaker, R. Krumme, S. C. Sweeney, and J. R. Hayes Jr., "Structural control of building response using shape-memory alloys: phase 1," Technical Report 95/22, U. S. Army Corps of Engineers Research Laboratories, Champaign, IL, USA, 1995.

[3] M. Dolce, D. Cardone, and R. Marnetto, "Implementation and testing of passive control devices based on shape memory alloys," Earthquake Engineering and Structural Dynamics, vol. 29, no. 7, pp. 945-968, 2000.

[4] D. J. Pines, "Real-time seismic damping and frequency control of steel structures using nitinol wire," Proceedings of SPIE-The International Society for Optical Engineering, vol. 4696, pp. 176-185, 2002.

[5] Y. Zhang and S. Zhu, "A shape memory alloy-based reusable hysteretic damper for seismic hazard mitigation," Smart Materials and Structures, vol. 16, no. 5, pp. 1603-1613, 2007.

[6] Y.-L. Han, Q. S. Li, A.-Q. Li, A. Y. T. Leung, and P.-H. Lin, "Structural vibration control by shape memory alloy damper," Earthquake Engineering and Structural Dynamics, vol. 32, no. 3, pp. 483-494, 2003.

[7] G. Song, N. Ma, and H. N. Li, "Applications of shape memory alloys in civil structures," Engineering Structures, vol. 28, no. 9, pp. 1266-1274, 2006.

[8] A. Abolmaali, J. Treadway, P. Aswath, F. K. Lu, and E. Mccarthy, "Hysteresis behavior of t-stub connections with superelastic shape memory fasteners," Journal of Constructional Steel Research, vol. 62, no. 8, pp. 831-838, 2006.

[9] H. Ma, T. Wilkinson, and C. Cho, "Feasibility study on a selfcentering beam-to-column connection by using the superelastic behavior of SMAs," Smart Materials and Structures, vol. 16, no. 5, pp. 1555-1563, 2007.

[10] H. Ma, T. Wilkinson, and C. Cho, "A numerical study on bolted end-plate connection using shape memory alloys," Materials and Structures, vol. 41, no. 8, pp. 1419-1426, 2008.

[11] C. Fang, M. C. H. Yam, A. C. C. Lam, and L. Xie, "Cyclic performance of extended end-plate connections equipped with shape memory alloy bolts," Journal of Constructional Steel Research, vol. 94, pp. 122-136, 2014.

[12] M. S. Speicher, R. DesRoches, and R. T. Leon, "Experimental results of a NiTi shape memory alloy (SMA)-based recentering beam-column connection," Engineering Structures, vol. 33, no. 9, pp. 2448-2457, 2011.

[13] W. Wang, T. M. Chan, H. Shao, and Y. chen, "Cyclic behavior of connections equipped with NiTi shape memory alloy and steel tendons between H-shaped beam to CHS column," Engineering Structures, vol. 88, pp. 37-50, 2015.

[14] X. Xu, Y. Zhang, and Y. Luo, "Self-centering modularized link beams with post-tensioned shape memory alloy rods," Engineering Structures, vol. 112, pp. 47-59, 2016.

[15] C. Fang, W. Wang, C. He, and Y. Y. Chen, "Self-centring behaviour of steel and steel-concrete composite connections equipped with NiTi SMA bolts," Engineering Structures, vol. 150, pp. 390-408, 2017.

[16] R. Desroches, J. McCormick, and M. Delemont, "Cyclic properties of superelastic shape memory alloy wires and bars," Journal of Structural Engineering, vol. 30, no. 1, pp. 38-46, 2004.

[17] C. Fang, M. C. H. Yam, H. Ma, and K. F. Chung, "Tests on superelastic Ni-Ti SMA bars under cyclic tension and directshear: towards practical recentring connections," Materials and Structures, vol. 48, no. 4, pp. 1013-1030, 2013.

[18] W. Wang, C. Fang, and J. Liu, "Large size superelastic SMA bars: heat treatment strategy, mechanical property and seismic application," Smart Materials and Structures, vol. 25, no. 7, p. 075001, 2016.
[19] F. Auricchio, and R. L. Taylor, "Shape-memory alloys: modeling and numerical simulations of the finite-strain superelastic behavior," Computer Methods in Applied Mechanics and Engineering, vol. 143, no. 1-2, pp. 175-194, 1997.

[20] F. Auricchio, R. L. Taylor, and J. Lubliner, "Shape-memory alloys: macromodelling and numerical simulations of the superelastic behavior," Computer Methods in Applied Mechanics and Engineering, vol. 146, no. 3-4, pp. 281-312, 1997.

[21] X. Xu, Y. Zhang, and Y. Luo, "Self-centering eccentrically braced frames using shape memory alloy bolts and posttensioned tendons," Journal of Constructional Steel Research, vol. 125, no. 10, pp. 190-204, 2016.

[22] M. C. H. Yam, F. Cheng, A. C. C. Lam, and Y. Zhang, "Numerical study and practical design of beam-to-column connections with shape memory alloys," Journal of Constructional Steel Research, vol. 104, pp. 177-192, 2015.

[23] AISC, Seismic Provisions for Structural Steel Buildings, ANSI/AISC 341-10, AISC, Chicago, IL, USA, 2010.

[24] Standardization Administration of The People's Republic of China, Hot Rolled Section Steel, GB/T 706-2008, Standardization Administration of The People's Republic of China, Beijing, China, 2008.

[25] N. Mansour, Eccentrically Braced Frames with Replaceable Shear Links, University of Toronto, Toronto, ON, Canada, 2010.

[26] N. Mansour, C. Christopoulos, and R. Tremblay, "Experimental validation of replaceable shear links for eccentrically braced steel frames," Journal of Structural Engineering, vol. 137, no. 10, pp. 1141-1152, 2011.

[27] ANSYS, Inc., ANSYS v.12.1, ANSYS, Inc., Canonsburg, PA, USA, 2009. 


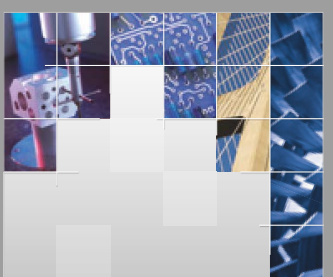

\section{Enfincering}
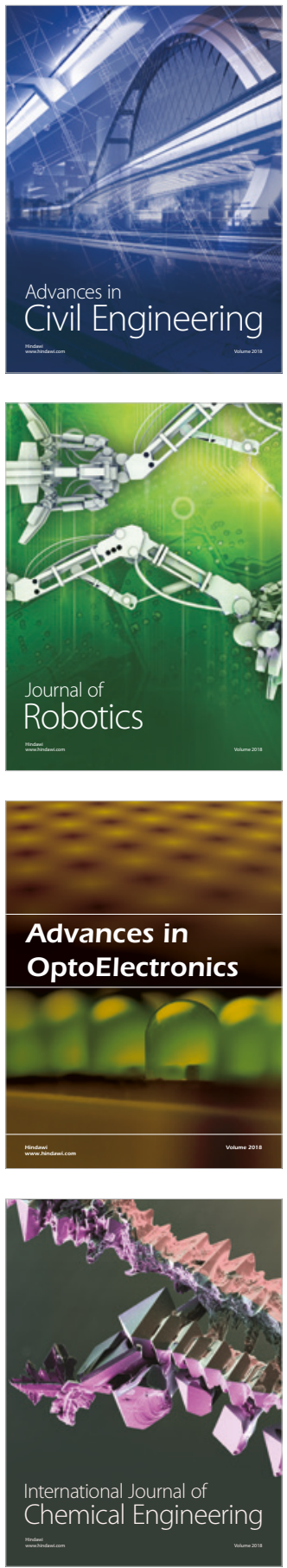

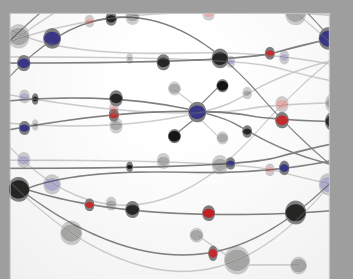

\section{Rotating \\ Machinery}

The Scientific World Journal

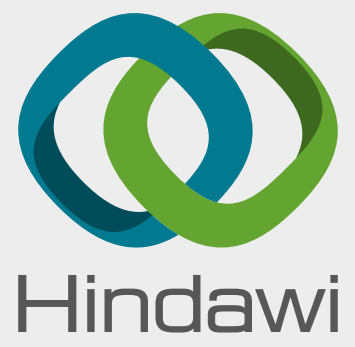

Submit your manuscripts at

www.hindawi.com
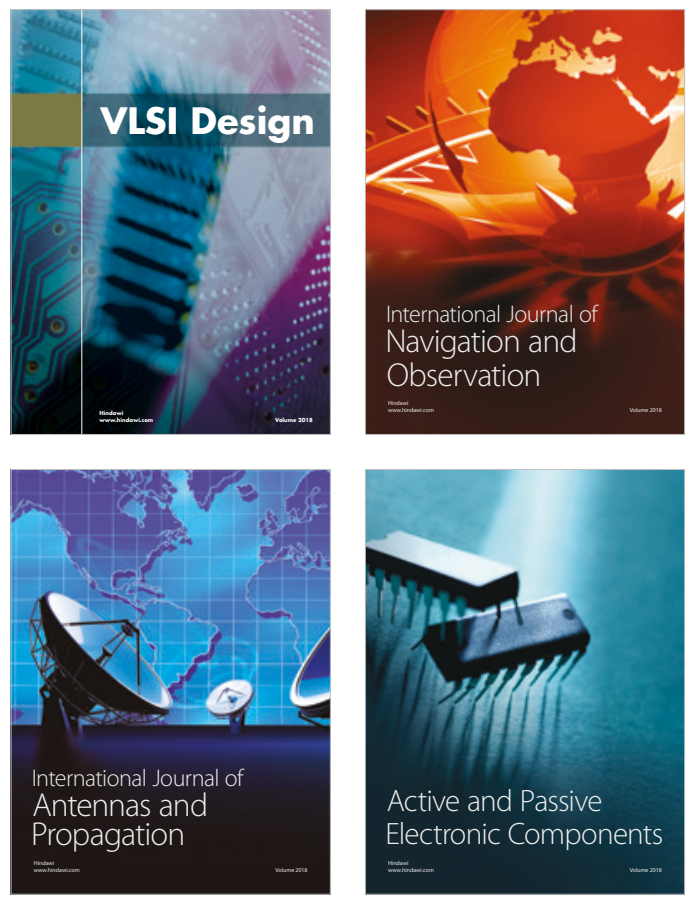
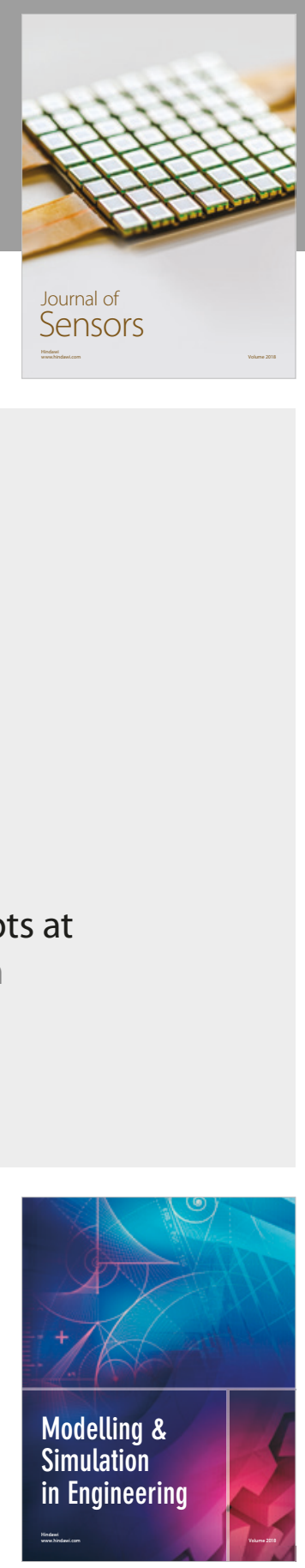

\section{Advances \\ Multimedia}
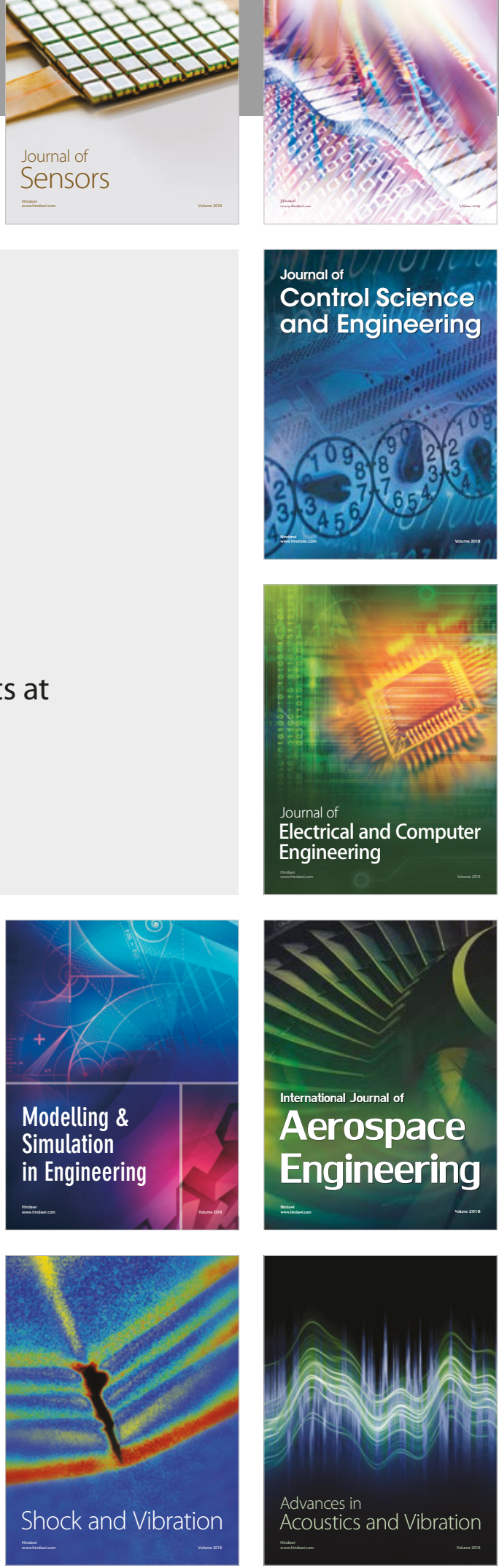